records of natural products

\title{
Triterpenoids from Acokanthera schimperi in Ethiopia
}

\author{
Wondwonsen A. Matebie $\odot^{1,2}$, Wanchang Zhang ${ }^{1}$, Shuo Zhang ${ }^{1}$ \\ and Guangbo Xie ${ }^{1 *}$

\begin{abstract}
${ }^{I}$ School of Life Science and Technology; Center for Informational Biology, University of Electronic
Science and Technology of China, Chengdu 610054, Sichuan, China

${ }^{2}$ Department of Chemical Engineering, Hawassa University Institute of Technology, Hawassa
\end{abstract} \\ P.O.Box 05, Ethiopia
}

(Received July 11, 2018; Revised August 21, 2018; Accepted September 18, 2018)

\begin{abstract}
The studies on the leaves of Acokanthera schimperi, a traditional herb of Ethiopia, afforded eight triterpenoids, including a new triterpenoid ester, lupan-20-ol-3( $\beta$ )-yl 3-hydroxyoctadecanoate (1), along with seven known triterpenoids, lupeol (2), 28-nor-urs-12-ene-3 $\beta, 17 \beta$-diol (3), ursolic aldehyde (4), 3 $\beta$-hydroxyoleana-11,13(18)-dien-28-oic acid (5), alagidiol (6), oleanolic acid (7) and ursolic acid lactone (8). Their structures were determined by spectroscopic methods including 2D NMR techniques and X-ray diffraction analysis.
\end{abstract}

Keywords: Acokanthera schimperi; triterpenoids; Ethiopian medicinal plants; spectroscopic analyses; X-ray diffraction. (c) 2018 ACG Publications. All rights reserved.

\section{Introduction}

Acokanthera schimperi (A. DC.) Schweinf, belonging to the family Apocynaceae, was called "Merenz" locally. It is a well-known East African arrow poison plant and found from Eritrea south to Tanzania and west to Uganda, Rwanda and eastern DR Congo. It is also found in southern Yemen. $[1,2]$. In Ethiopia, the leaves and bark of $A$. schimperi were applied to the skin to treat skin disorders, and an infusion of its leaves was gargled to treat tonsillitis [2]. Ethiopian traditional healers also used this indigenous plant for the treatment of headache, epilepsy, amnesia, elephantiasis, scabies, leprosy, etc [3]. Literature survey revealed that most of the researches were focused on the bioactivities and traditional use of this plant [4-6], and less report was found for its phytochemical studies [7]. Recently, we carried out a systematic chemical study on the leaves of $A$. schimperi, and eight triterpenoids (1-8), including a new triterpenoid ester, lupan-20-ol-3( $\beta$ )-yl 3-hydroxyoctadecanoate (1), were isolated. Herein, we report the isolation, structural elucidation of these compounds.

\footnotetext{
*Corresponding author: E-Mail: gbxie@ uestc.edu.cn ; Phone:086-28-83208238 Fax:086-28-83208238 


\section{Materials and Methods}

\subsection{Instrumentation and Reagents}

Optical rotation was measured on APVI/6W polarimeter (Rudolph Research Analytical). IR spectrum was recorded on a Nicolet 6700 FT-IR spectrometer (ThermoFisher). HRESIMS data were acquired on a Water Q-TOF Premier. NMR spectra were obtained on a Varian NMR System 600 or Bruker AV II-600 and 400, with tetramethylsilane as an internal standard. X-ray diffraction data were collected on a Bruker Xcalibur E CCD diffractometer using graphite-monochromated Mo Ka radiation. Materials for column chromatography (CC) were silica gel (Qingdao Marine Chemical Factory, Qingdao, China) or Sephadex LH-20 (GE Healthcare, USA). Analytical and preparative thinlayer chromatography (TLC) was conducted on GF254 or G plates. All the chemical reagents and solvents used for separation and purification were analytical grade and purchased from local firms (Kelong chemical reagent factory, Chengdu, China).

\subsection{Plant Materials}

The leaves of A. schimperi were collected in the Debre Libanos monastery, lying northwest of Addis Ababa, Ethiopia. It was identified by Amare Seifu Assefa, a botanist from the Ethiopian Biodiversity Institute. A voucher specimen (AS-2015-10) was deposited in the Herbarium of Ethiopian Biodiversity Institute, Ethiopia.

\subsection{Extraction and Isolation}

At room temperature, the powdered leaves of A. schimperi $(3.35 \mathrm{~kg})$ were extracted with EtOH: $\mathrm{H}_{2} \mathrm{O}(4 \times 12 \mathrm{~L}, 95: 5 \mathrm{v} / \mathrm{v}, 7$ days each time $)$. The extracts were concentrated in vacuo to yield a residue that was suspended in $\mathrm{H}_{2} \mathrm{O}(7 \mathrm{~L})$ and extracted with EtOAc $(3 \times 7 \mathrm{~L})$. The EtOAc extract $(122$ g) was applied to CC over silica gel (200-300 mesh, $1.8 \mathrm{~kg}$ ) and eluted with cyclohexane-ethyl acetate (100:1-1:1, gradient system). On the basis of the TLC analysis, ten fractions A-J were obtained.

Fr. C $(1.1 \mathrm{~g})$ was isolated by silica gel chromatography using a solvent system cyclehexaneacetone (60:1) to get two subfractions (Fr. C-1 and C-2). Fr. C-1 was separated by slica gel chromatography (chloroform-cyclohexane-acetone, 60:80:1) and purified on Sephadex LH-20 (chloroform-methanol, 2:1) to yield $4(16 \mathrm{mg})$. Fr. C-2 was separated using preparative TLC (cyclohexane-acetone, 5:1) and purified by Sephadex LH 20 (chloroform-methanol, 2:1) to get 2 (22 $\mathrm{mg})$. Fr. D ( $0.9 \mathrm{~g})$ was isolated by silica gel chromatography (chloroform-cyclohexane-acetone, 30:50:1) and preparative TLC (cyclohexane-acetone, 6:1), and then purified by Sephadex LH-20 (chloroform-methanol, 2:1) to afford $3(18 \mathrm{mg})$. Fr. E $(0.6 \mathrm{~g})$ was separated by silica gel chromatography (chloroform-cyclohexane-acetone, 30:50:1) to afford two subfractions (Fr. E-1 and E2). Fr. E-1 was separated by silica gel chromatography (cyclohexane-acetone, 10:1) and purified with Sephadex LH-20 (chloroform-methanol, 2:1) to afford $5(8 \mathrm{mg}$ ). Fr. E-2 was isolated by silica gel chromatography (cyclohexane-acetone, 70:1) to give two subfractions (Fr. E-2-1 and E-2-2). Fr. E-2-1 was separated by silica gel chromatography (cyclohexane-acetone, 10:1) and purified using Sephadex LH-20 to afford 1 (9 mg). Fr. E-2-2 was separated by silica gel chromatography (cyclohexane-acetone, 13:1) and purified with Sephadex LH-20 to give $6(41 \mathrm{mg})$. Fr. F $(0.57 \mathrm{~g})$ was subjected to silica gel CC (chloroform-acetone, 150:1), and then purified by Sephadex LH-20 to give the 7 (7 mg). Fr. G $(1.47 \mathrm{~g})$ was isolated by silica gel chromatography (chloroform-acetone, 140:1; chloroform-ethyl acetate, 40:1) and purified with Sephadex LH-20 to afford 8 (8 mg).

Lupan-20-ol-3( $\beta$ )-yl 3-hydroxyoctadecanoate (1): white amorphous powder; $[\propto]_{D}^{20}:+5.97$ (c 0.067 , $\mathrm{CH}_{2} \mathrm{Cl}_{2}$ ); IR (KBr) $U_{\max } 3455,2923,2853,1714,1665,1381,1359,1172,979 \mathrm{~cm}^{-1} ;{ }^{1} \mathrm{H}-\mathrm{NMR}(600$ $\left.\mathrm{MHz}, \mathrm{CDCl}_{3}\right)$ and ${ }^{13} \mathrm{C}-\mathrm{NMR}\left(150 \mathrm{MHz}, \mathrm{CDCl}_{3}\right)$ spectral data, Table 1; HRESIMS (positive) $\mathrm{m} / \mathrm{z}$ $749.6432[\mathrm{M}+\mathrm{Na}]^{+}$(calcd for $\mathrm{C}_{48} \mathrm{H}_{86} \mathrm{O}_{4} \mathrm{Na}, 749.6424$ ). 
Alagidiol (6): colorless crystal $\left(\mathrm{CH}_{2} \mathrm{Cl}_{2}\right) ;{ }^{1} \mathrm{H}-\mathrm{NMR}\left(600 \mathrm{MHz}, \mathrm{CDCl}_{3}\right) \delta_{\mathrm{H}}: 3.20(1 \mathrm{H}, \mathrm{dd}, J=11.5,4.7$ $\mathrm{Hz}, \mathrm{H}-3), 1.11,0.99,0.97,0.84,0.84,0.76$ (each $\left.3 \mathrm{H}, \mathrm{s}, 6 \times \mathrm{CH}_{3}\right), 0.97\left(3 \mathrm{H}, \mathrm{d}, J=7.0 \mathrm{~Hz}, \mathrm{CH}_{3}\right), 0.85$ $\left(3 \mathrm{H}, \mathrm{d}, J=6.6 \mathrm{~Hz}, \mathrm{CH}_{3}\right) .{ }^{13} \mathrm{C}-\mathrm{NMR}\left(150 \mathrm{MHz}, \mathrm{CDCl}_{3}\right) \delta_{\mathrm{C}}: 83.6(\mathrm{C}-18), 78.9(\mathrm{C}-3), 55.3(\mathrm{C}-5), 50.7$ (C-9), 45.9 (C-21), 45.8 (C-17), 41.4 (C-14), 41.1 (C-8), 39.4 (C-13), 38.8 (C-4), 38.7 (C-1), 37.1 (C10), 35.0 (C-16), 32.9 (C-7), 30.8 (C-22), 28.2 (C-20), 27.9 (C-23), 27.3 (C-2), 25.7 (C-15 or C-19), 24.8 (C-12), 22.7 (C-29), 22.7 (C-30), 21.9 (C-15 or C-19), 21.4 (C-11), 18.3 (C-6), 17.8 (C-28), 16.3 (C-24), 16.0 (C-27), 15.9 (C-25), 15.3 (C-26). HRESIMS (positive) $\mathrm{m} / z 467.3860$ [M+Na] ${ }^{+}$(calcd for $\left.\mathrm{C}_{30} \mathrm{H}_{52} \mathrm{O}_{2} \mathrm{Na}, 467.3865\right)$.

\subsection{X-ray Crystallographic Study}

Alagidiol (6) (Figure 1): $\mathrm{C}_{30} \mathrm{H}_{52} \mathrm{O}_{2}, M=444.71, T=293 \mathrm{~K}, \lambda=0.71073 \AA$, orthorhombic, $\mathrm{P} 2(1) 2(1) 2, a=19.6011$ (9) $\AA, b=19.5010$ (7) $\AA, c=7.7285$ (3) $\AA, V=2954.2$ (2) $\AA^{3}, Z=4 . D_{\mathrm{c}}=$ $1.000 \mathrm{~g} \mathrm{~cm}^{-3}, \mathrm{Mu}=0.060 \mathrm{~mm}^{-1}, F(000)=992$. Data were collected using a colorless block of size 0.35 $\times 0.30 \times 0.25 \mathrm{~mm}$ in the range $2.95^{\circ} \leq \theta \leq 26.37^{\circ}$ within the index range $-24 \leq h \leq 21,-24 \leq k \leq 16,-9$ $\leq l \leq 9.9498$ reflections measured, 5461 unique reflections, $R_{\text {int }}=0.0199$. Refinement by full-matrix least-squares on $F^{2}$ converged to give final $R$ indices $R_{1}=0.0522, w R_{2}=0.1175[I>2 \sigma(I)]$ and $R_{1}=$ $0.0701, w R_{2}=0.1258$ (all data). Data/restraints/parameters $=5461 / 8 / 313$, goodness-of-fit on $F^{2}=$ 1.046 , largest difference peak and hole are 0.14 and -0.16 e $\AA^{-3}$. Crystallographic data for 6 have been deposited with the Cambridge Crystallographic Data Center as supplementary publication number CCDC 1854896. These data can be obtained free of charge via www.ccdc.cam.ac.uk/deposit (or from the CCDC, 12 Union Road, Cambridge CB2 1EZ, UK; fax: +44 1223 336033; deposit@ccdc.cam.ac.uk).

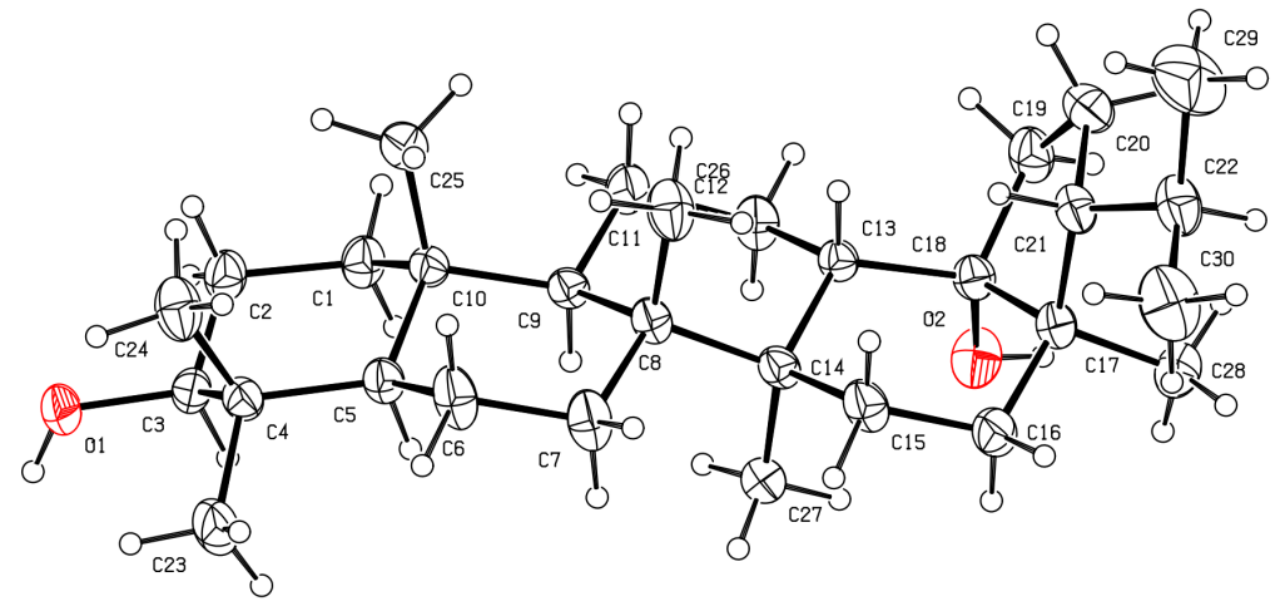

Figure 1. X-ray ctystal structure of compound 6

\section{Results and Discussion}

Phytochemical research was performed for the leaves of $A$. schimperi by chromatography and afforded a new triterpenoid ester, lupan-20-ol-3( $\beta$ )-yl 3-hydroxyoctadecanoate (1), together with seven known triterpenoids, namely lupeol (2) [9], 28-nor-urs-12-ene-3 $\beta, 17 \beta$-diol (3) [10], ursolic aldehyde (4) [8], 3 $\beta$-hydroxy-oleana-11,13(18)-dien-28-oic acid (5) [11], alagidiol (6) [12,13], oleanolic acid (7) [14] and ursolic acid lactone (8) [15] (Figure 2). The structures of the known compounds were identified by comparing their spctroscopic data with those reported in the literatures.

Compound 1 was isolated as white amorphous powder. Its molecular formula was determined to be $\mathrm{C}_{48} \mathrm{H}_{86} \mathrm{O}_{4}$ on the basis of HRESIMS $\left(\mathrm{m} / z\right.$ : $[\mathrm{M}+\mathrm{Na}]^{+}$calcd 749.6424 ; found 749.6432$)$ and its ${ }^{13} \mathrm{C}-$ NMR spectroscopic data (Table 1), indicated 6 degrees of unsaturation. 


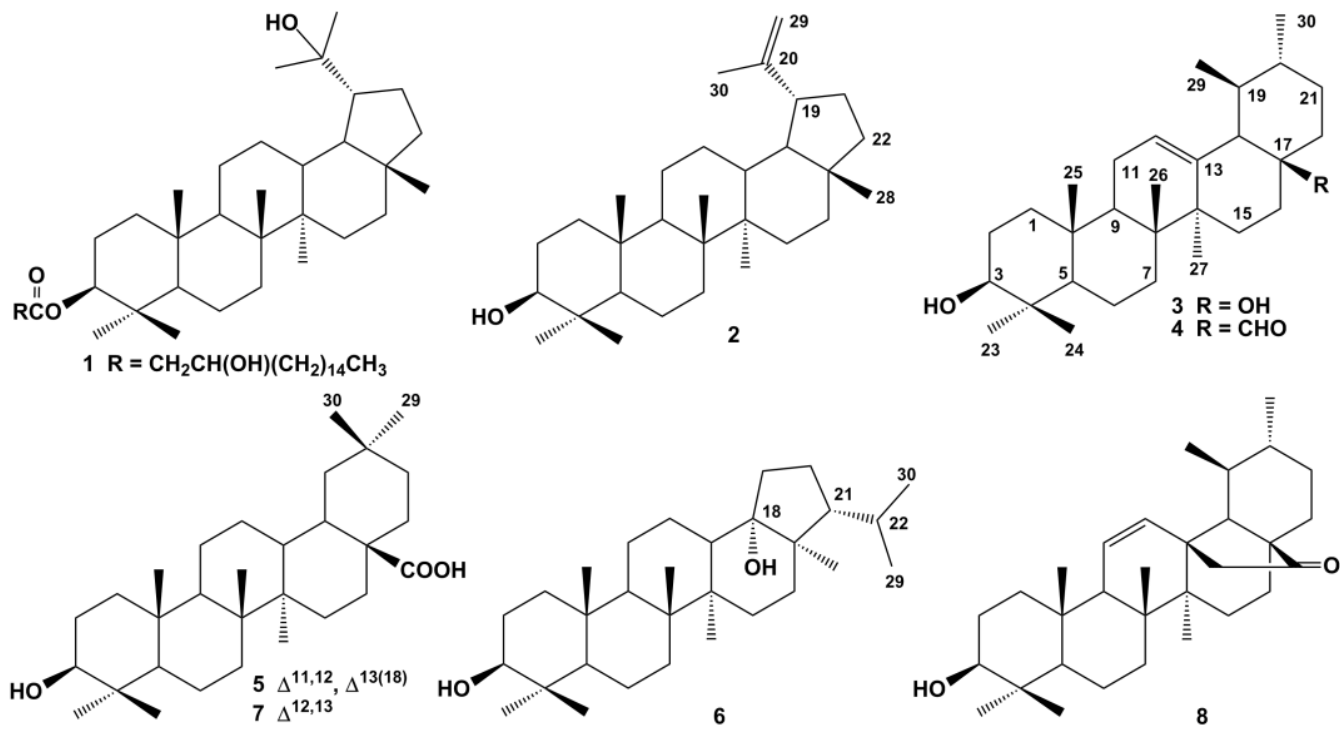

Figure 2. Structures of compounds 1-8

The IR absorptions indicated the presence of hydroxyl group $\left(3455 \mathrm{~cm}^{-1}\right)$ and carbonyl group $\left(1714 \mathrm{~cm}^{-1}\right)$. Analysis of the ${ }^{1} \mathrm{H}$ and ${ }^{13} \mathrm{C}$ NMR spectra of 1 (Table 1) showed a set of triterpenoid signals, eight tertiary methyl groups $\left[\delta_{\mathrm{H}} 1.22(\mathrm{~s}), \delta_{\mathrm{C}} 31.9 ; \delta_{\mathrm{H}} 1.12(\mathrm{~s}), \delta_{\mathrm{C}} 24.7 ; \delta_{\mathrm{H}} 1.05(\mathrm{~s}), \delta_{\mathrm{C}} 16.1 ; \delta_{\mathrm{H}}\right.$ $\left.0.95(\mathrm{~s}), \delta_{\mathrm{C}} 14.8 ; \delta_{\mathrm{H}} 0.86(\mathrm{~s}), \delta_{\mathrm{C}} 16.2 ; \delta_{\mathrm{H}} 0.85(\mathrm{~s}), \delta_{\mathrm{C}} 28.0 ; \delta_{\mathrm{H}} 0.84(\mathrm{~s}), \delta_{\mathrm{C}} 16.6 ; \delta_{\mathrm{H}} 0.80(\mathrm{~s}), \delta_{\mathrm{C}} 19.2 ;\right]$, ten methylene groups $\left[\delta_{\mathrm{H}} 1.00(\mathrm{~m}), 1.68(\mathrm{~m}), \delta_{\mathrm{C}} 38.3 ; \delta_{\mathrm{H}} 1.27(\mathrm{~m}), 1.63(\mathrm{~m}), \delta_{\mathrm{C}} 23.7 ; \delta_{\mathrm{H}} 1.40(\mathrm{~m}), 1.51\right.$ $(\mathrm{m}), \delta_{\mathrm{C}} 18.2 ; \delta_{\mathrm{H}} 1.40(\mathrm{~m}), \delta_{\mathrm{C}} 34.4 ; \delta_{\mathrm{H}} 1.26(\mathrm{~m}), 1.47(\mathrm{~m}), \delta_{\mathrm{C}} 21.4 ; \delta_{\mathrm{H}} 1.11(\mathrm{~m}), 1.76(\mathrm{~m}), \delta_{\mathrm{C}} 27.5 ; \delta_{\mathrm{H}}$ $1.32(\mathrm{~m}), 1.62(\mathrm{~m}), \delta_{\mathrm{C}} 28.7 ; \delta_{\mathrm{H}} 1.34(\mathrm{~m}), 1.50(\mathrm{~m}), \delta_{\mathrm{C}} 35.5 ; \delta_{\mathrm{H}} 1.60(\mathrm{~m}), 1.87(\mathrm{~m}), \delta_{\mathrm{C}} 28.9 ; \delta_{\mathrm{H}} 1.10(\mathrm{~m})$, $\left.1.31(\mathrm{~m}), \delta_{\mathrm{C}} 40.1\right]$, six methine groups $\left[\delta_{\mathrm{H}} 4.54(\mathrm{dd}, 10.0,6.24), \delta_{\mathrm{C}} 81.4 ; \delta_{\mathrm{H}} 0.79(\mathrm{~m}), \delta_{\mathrm{C}} 55.2 ; \delta_{\mathrm{H}} 1.27\right.$ $\left.(\mathrm{m}), \delta_{\mathrm{C}} 50.1 ; \delta_{\mathrm{H}} 1.71(\mathrm{~m}), \delta_{\mathrm{C}} 37.4 ; \delta_{\mathrm{H}} 1.33(\mathrm{~m}), \delta_{\mathrm{C}} 48.2 ; \delta_{\mathrm{H}} 1.79(\mathrm{~m}), \delta_{\mathrm{C}} 49.9\right]$ and six quaternary carbons $\left(\delta_{\mathrm{C}} 73.4,44.6,43.5,41.3,37.7,36.9\right)$. These NMR data were matching with the triterpenoid

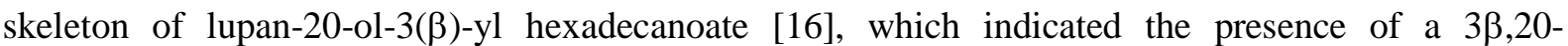
dihydroxylupane skeleton for $\mathbf{1}$. In addition to the signals for triterpenoid, signals for a saturated fatty acid were also found, one $\mathrm{CH}_{3}\left[\delta_{\mathrm{H}} 0.88(\mathrm{t}, 6.8), \delta_{\mathrm{C}} 14.1\right]$, one oxygen-bearing $\mathrm{CH}\left[\delta_{\mathrm{H}} 3.99(\mathrm{~m}), \delta_{\mathrm{C}}\right.$ 68.2], fifteen $\mathrm{CH}_{2}\left[\delta_{\mathrm{H}} 2.50(\mathrm{dd}, 16.2,2.9), 2.39(\mathrm{dd}, 16.2,9.0), \delta_{\mathrm{C}} 41.6 ; \delta_{\mathrm{H}} 1.25 \sim 1.50(\mathrm{~m}, 28 \mathrm{H}), \delta_{\mathrm{C}}\right.$ $36.5,25.4,29.3 \sim 31.5(11 \mathrm{C}), 22.7]$ and one carbonyl quaternary carbon $\left(\delta_{\mathrm{C}} 172.7\right)$, which was similar with the NMR signals of the fatty acid moiety of methyl 3-hydroxyoctadecanoate and 2-O-[(R)-3hydroxyhexadecanoyl]glycerol $[17,18]$. Compared with 3 $\beta, 20$-dihydroxylupane, the downfield of $\mathrm{H}-3$ $(+1.34), \mathrm{C}-3(+2.4)$ and upfield of C-2 (-3.9) of $\mathbf{1}$ indicated that the fatty acid was esterified with $3-\mathrm{OH}$ $[19,20]$, which was confirmed by the correlation of $\delta_{\mathrm{H}} 4.54(\mathrm{H}-3)$ and $\delta_{\mathrm{C}} 172.7\left(\mathrm{C}-1{ }^{\prime}\right)$ in the HMBC spectrum (Figure 3). Thus, the structure of compound $\mathbf{1}$ was elucidated and named as lupan-20-ol3( $\beta)$-yl 3-hydroxyoctadecanoate. 


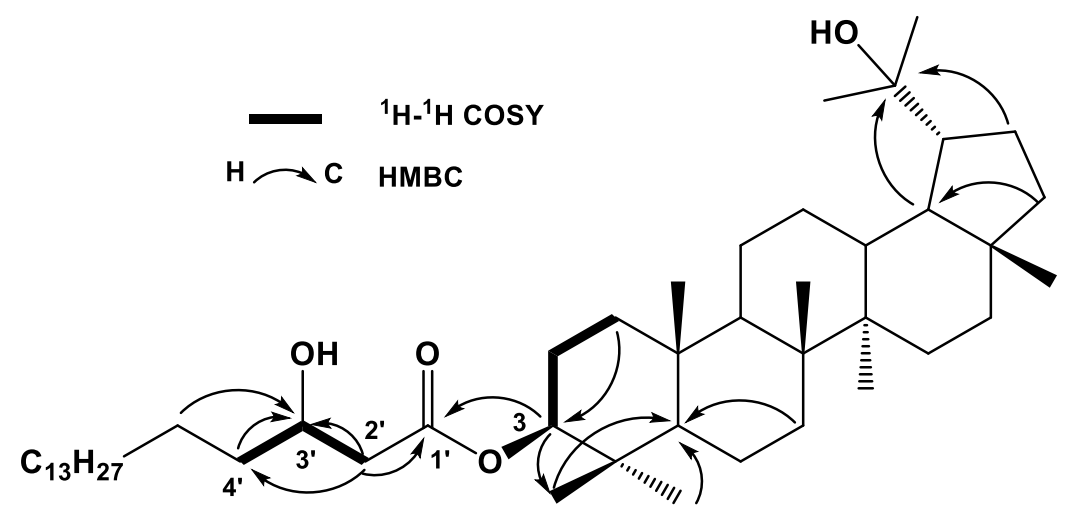

Figure 3. Selected ${ }^{1} \mathrm{H}_{-}{ }^{1} \mathrm{H}$ COSY and HMBC correlation of compound 1

Table 1. ${ }^{1} \mathrm{H}$ and ${ }^{13} \mathrm{C}$ NMR data for $\mathbf{1}\left(600\right.$ and $150 \mathrm{MHz}$ in $\mathrm{CDCl}_{3}, \delta$ in ppm, $J$ in $\left.\mathrm{Hz}\right)$.

\begin{tabular}{|c|c|c|c|c|c|}
\hline Position & $\delta_{\mathrm{H}}($ mult., $J$ in $\mathrm{Hz})$ & $\delta_{\mathrm{C}}$ & Position & $\delta_{\mathrm{H}}$ (mult., $J$ in $\left.\mathrm{Hz}\right)$ & $\delta_{\mathrm{C}}$ \\
\hline 1 & $1.00(1 \mathrm{H}, \mathrm{m}) ; 1.68(1 \mathrm{H}, \mathrm{m})$ & 38.3 & 20 & & 73.4 \\
\hline 2 & $1.27(1 \mathrm{H}, \mathrm{m}) ; 1.63(1 \mathrm{H}, \mathrm{m})$ & 23.7 & 21 & $1.60(1 \mathrm{H}, \mathrm{m}) ; 1.87(1 \mathrm{H}, \mathrm{m})$ & 28.9 \\
\hline 3 & $4.54(1 \mathrm{H}, \mathrm{dd}, 6.24,10.0)$ & 81.4 & 22 & $1.10(1 \mathrm{H}, \mathrm{m}) ; 1.31(1 \mathrm{H}, \mathrm{m})$ & 40.1 \\
\hline 4 & & 37.7 & 23 & $0.85(3 \mathrm{H}, \mathrm{s})$ & 28.0 \\
\hline 5 & $0.79(1 \mathrm{H}, \mathrm{m})$ & 55.2 & 24 & $0.84(3 \mathrm{H}, \mathrm{s})$ & 16.6 \\
\hline 6 & $1.40(1 \mathrm{H}, \mathrm{m}) ; 1.51(1 \mathrm{H}, \mathrm{m})$ & 18.2 & 25 & $0.86(3 \mathrm{H}, \mathrm{s})$ & 16.2 \\
\hline 7 & $1.40(2 \mathrm{H}, \mathrm{m})$ & 34.4 & 26 & $1.05(3 \mathrm{H}, \mathrm{s})$ & 16.1 \\
\hline 8 & & 41.3 & 27 & $0.95(3 \mathrm{H}, \mathrm{s})$ & 14.8 \\
\hline 9 & $1.27(1 \mathrm{H}, \mathrm{m})$ & 50.1 & 28 & $0.80(3 \mathrm{H}, \mathrm{s})$ & 19.2 \\
\hline 10 & & 36.9 & 29 & $1.12(3 \mathrm{H}, \mathrm{s})$ & 24.7 \\
\hline 11 & $1.47(1 \mathrm{H}, \mathrm{m}) ; 1.26(1 \mathrm{H}, \mathrm{m})$ & 21.4 & 30 & $1.22(3 \mathrm{H}, \mathrm{s})$ & 31.9 \\
\hline 12 & $1.11(1 \mathrm{H}, \mathrm{m}) ; 1.76(1 \mathrm{H}, \mathrm{m})$ & 27.5 & 11 & & 172.7 \\
\hline 13 & $1.71(1 \mathrm{H}, \mathrm{m})$ & 37.4 & 21 & $\begin{array}{l}2.39(1 \mathrm{H}, \mathrm{dd}, 9.0,16.2) \\
2.50(1 \mathrm{H}, \mathrm{dd}, 2.9,16.2)\end{array}$ & 41.6 \\
\hline 14 & & 43.5 & 31 & $3.99(1 \mathrm{H}, \mathrm{m})$ & 68.2 \\
\hline 15 & $1.32(1 \mathrm{H}, \mathrm{m}) ; 1.62(1 \mathrm{H}, \mathrm{m})$ & 28.7 & 41 & & 36.5 \\
\hline 16 & $1.34(1 \mathrm{H}, \mathrm{m}) ; 1.50(1 \mathrm{H}, \mathrm{m})$ & 35.5 & 51 & 125,150 (2QU $\mathrm{m}$ & 25.4 \\
\hline 17 & & 44.6 & $6^{\prime}-16{ }^{\prime}$ & $1.25 \sim 1.50(20 \mathrm{H}, \mathrm{m})$ & $29.3 \sim 31.5$ \\
\hline 18 & $1.33(1 \mathrm{H}, \mathrm{m})$ & 48.2 & $17{ }^{\prime}$ & & 22.7 \\
\hline 19 & $1.79(1 \mathrm{H}, \mathrm{m})$ & 49.9 & $18^{\prime}$ & $0.88(3 \mathrm{H}, \mathrm{t}, 6.8)$ & 14.1 \\
\hline
\end{tabular}

Acokanthera belongs to the family Apocynaceae and it comprises 5 species, A. laevigata, A. oblongifolia, A. oppositifolia, A. rotundata and A. schimperi. Previous phytochemical studies on this genus were focused on the toxic cardiac glycosides, which were correlated with its usage as arrow poisons [21, 22]. But, besides cardenolides, less other type natural products were identified from genus Acokanthera. Karawya reported the isolation of three pentacyclic triterpenoids, friedelin, lupeol and $\beta$ amyrin, from A. spectabilis (also known as A. oblongifolia [23]) [24]. From A. oppositifolia, a pentacyclic triterpenoid, lupeol, and its ester, lup-20(29)-en-3 $\beta-O$-(3'- $\beta$-hydroxy) palmitate, together with $\beta$-sitosterol, have been isolated [25]. In our present study on A. schimperi, eight pentacyclic triterpenoids have been isolated, and they can be divided into four triterpene skeleton types, oleanane, ursane, lupane and hopane, which indicated the high structural diversity of triterpene in A. schimperi.

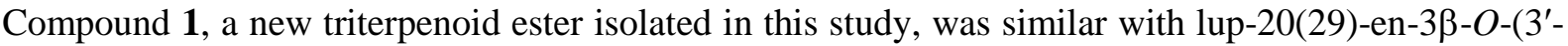
$\beta$-hydroxy) palmitate, a lupane ester from A. spectabilis [25]. Due to the lack of phytochemical data of this genus, the importance of triterpeoid in the chemotaxonomy of genus Acokanthera need further studied. 


\section{Acknowledgments}

This work was financially supported by the Fundamental Research Funds for the Central Universities (ZYGX2016J120). The authors thanked Dr. Haokun Yuan, School of Life Science and Technology, University of Electronic Science and Technology of China, for his kind help in crystallization of compound $\mathbf{6}$.

\section{Supporting Information}

Supporting Information accompanies this paper on http://www.acgpubs.org/journal/recordsof-natural-products

\section{ORCID}

Wondwonsen A. Matebie: 0000-0002-4667-7899

Wangchang Zhang: 0000-0003-0103-8476

Shuo Zhang: 0000-0002-5612-2256

Guangbo Xie: $\underline{0000-0001-7600-3214}$

\section{References}

[1] A. J. M. Leeuwenberg (2003). Apocynaceae, In: Flora of Ethiopia and Eritrea, Volume 4, Part 1, ed: I. Hedberg, S. Edwards and S. Nemomissa, The National Herbarium, Addis Ababa University, Addis Ababa, Ethiopia and Uppsala, Sweden, pp.87-88.

[2] G. H. Schmelzer and A. Gurib-Fakim, eds. (2008). Plant resources of tropical Africa 11(1). Medicinal plants 1, PORTA Foundation, Wageningen, Netherlands / Backhuys Publishers, Leiden, Netherlands / CTA, Wageningen, Netherlands, pp.32-34.

[3] D. Abebe and A. Ayehu (1993). Medicinal plants and enigmatic health practices of northern Ethiopia, Addis Ababa, Ethiopia.

[4] H. Tadeg, E. Mohammed, K. Asres and T. Gebre-Mariam (2005). Antimicrobial activities of some selected traditional Ethiopian medicinal plants used in the treatment of skin disorders, $J$. Ethnopharmacol. 100, 168-175.

[5] M. Giday, T. Teklehaymanot, A. Animut and Y. Mekonnen (2007). Medicinal plants of the Shinasha, Agew-awi and Amhara peoples in northwest Ethiopia, J. Ethnopharmacol. 110, 516-525.

[6] T. Mohammed, B. Erko and M. Giday (2014). Evaluation of antimalarial activity of leaves of Acokanthera schimperi and Croton macrostachyus against Plasmodium berghei in Swiss albino mice, BMC Complement. Altern. Med. 14, 314.

[7] J. O. Owino, J. C. Matasyoh and A. Y. Guliye (2015). Acaricidal coumarins from the medicinal plant Acokanthera schimperi, J. Org. Inorg. Chem. 1, 3.

[8] D. H. Kim, K. M. Han, I. S. Chung, D. K. Kim, S. H. Kim, B. M. Kwon, T. S. Jeong, M. H. Park, E. M. Ahn and N. I. Baek (2005). Triterpenoids from the flower of Campsis grandiflora K. Schum. as human acyl-CoA: cholesterol acyltransferase inhibitors, Arch. Pharm. Res. 28, 550-556.

[9] Y. M. Luo, A. H. Liu, B. W. Yu, L. J. Kang and L. Q. Huang (2005). Studies on chemical constituents of Sarcandra glabra, Chin. Pharm. J. 40, 1296-1298.

[10] M. Zhao, S. Zhuang, L. Fu, N. Li, J. Ba, J. Sakai, L. Wang, W. Tang, T. Hasegawa, H. Ogura, T. Kataoka, S. Oka, M. Kiuch, K. Hirose and M. Ando (2006). Taraxasterane- and ursane-type triterpenes from Nerium oleander and their biological activities, J. Nat. Prod. 69, 1164-1167.

[11] A. Ikuta, K. Kamiya, T. Satake and Y. Saiki (1995). Triterpenoids from callus tissue cultures of Paeonia species, Phytochemistry 38, 1203-1207.

[12] B. Achari, A. Pal and S. C. Pakrashi (1975). Studies on Indian medicinal plants. Part XXXVI: new D:Ecis neohopane derivatives from Alangium lamarckii, Tetrahedron Lett. 48, 4275-4278.

[13] M. J. U. Ferreira, A. M. Lobo and H. Wyler (1993). Triterpenoids and steroids from Euphorbia replus, Fitoterapia 64, 85-87.

[14] J. Tian, G. B. Xie, Y. Xie and T. N. Li (2015). Terpenoids from Ligularia kangtingensis, Phcog. Mag. 11, 44-47.

[15] H. Wang and Y. Fujimoto (1993). Triterpene esters from Eucalyptus tereticornis, Phytochemistry 33, 151-153. 
[16] R. Rajan, R. Venkataraman and S. Baby (2016). A new lupane-type triterpenoid fatty acid ester and other isolates from Ophiorrhiza shendurunii, Nat. Prod. Res. 30, 2197-2203.

[17] C. Feichter, K. Faber and H. Griengl (1990). Chemoenzymatic preparation of optically active longchain 3-hydroxyalkanoates, Biocatal. 3, 145-158.

[18] A. Okawa, K. Ohyama and Y. Fujimoto (2013). 2-O-(3-hydroxyhexadecanoyl)glycerol and 2-O-(3hydroxytetradecanoyl)glycerol and their 1-O-acetyl derivatives from the glandular trichome exudate of Verbascum blattaria f. erubescens, Nat. Prod. Res. 27, 1372-1377.

[19] W. H. Hui and M. M. Li (1977). Further triterpenoids from the stems of Lithocarpus polystachya, Phytochemistry 16, 111-112.

[20] A. Yürüker, J. Orjala, O. Sticher and T. Rali (1998). Triterpene from Rhus taitensis, Phytochemistry 48, 863-866.

[21] A. A. Adedapo, F. O. Jimoh, A. J. Afolayan and P. J. Masika (2008). Antioxidant activities and phenolic contents of the methanol extracts of the stems of Acokanthera oppositifolia and Adenia gummifera, BMC Complement. Altern. Med. 8, 54.

[22] A. G. Hanna, M. H. A. Elgamal, A. Z. Hassan, H. Duddeck, A. Simon, J. Kovács and G. Tóth (1998). Complete ${ }^{1} \mathrm{H}$ and ${ }^{13} \mathrm{C}$ signal assignments of $5 \beta$-cardenolides isolated from Acokanthera spectabilis Hook F., Magn. Reson. Chem. 36, 936-942.

[23] F. K. Kupicha (1982). Studies on African Apocynaceae: the genus Acokanthera, Kew Bulletin 37, 41-67.

[24] M. S. Karawya, S. M. Abdel-Wahab and H. M. Niazi (1973). Preliminary phytochemical screening of Acokanthera spectabilis, Planta Med. 24, 234-242.

[25] A. M. El Sayed, S. M. Ezzat and O. M. Sabry (2016). A new antibacterial lupane ester from the seeds of Acokanthera oppositifolia Lam., Nat. Prod. Res. 30, 2813-2818.

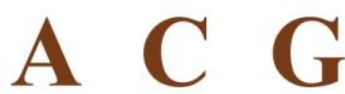

publications

(C) 2019 ACG Publications 